\title{
Analysis of postoperative complications and risk factors in patients with permanent pacemaker implantation
}

\author{
Songbo Jing, Shan Hu, Shuai Ma \\ Department of Cardiovascular Medicine, Affiliated Hospital of Zunyi Medical University, Zunyi, China \\ Contributions: (I) Conception and design: S Jing; (II) Administrative support: S Hu; (III) Provision of study materials or patients: S Jing, S Hu; (IV) \\ Collection and assembly of data: All authors; (V) Data analysis and interpretation: S Jing, S Ma; (VI) Manuscript writing: All authors; (VII) Final \\ approval of manuscript: All authors. \\ Correspondence to: Songbo Jing. Department of Cardiovascular Medicine, Affiliated Hospital of Zunyi Medical University, 149 Dalian Road, Huichuan \\ District, Zunyi 563003, China. Email: 18108529583@163.com.
}

\begin{abstract}
Background: Permanent pacemaker implantation (PPI) is widely used for the treatment of chronic arrhythmia. The aim of the present study was to investigate the incidence of postoperative complications and the risk factors in patients with PPI.

Methods: The clinical data of 124 patients who underwent PPI in our hospital from January 2014 to January 2018 were collected and analyzed retrospectively. Data on sex, age, status of disease, and related complications of all patients were recorded, and differences were compared. The factors affecting postoperative complications in patients with PPI were analyzed.

Results: The incidence of postoperative complications in 124 patients with PPI was 8.06\% (10/124), including 4 cases with capsular hematoma (3.23\%), 2 cases with capsular rupture (1.61\%), 3 cases with capsular infection $(2.42 \%)$ and 1 case with venous thrombosis $(0.81 \%)$. Unconditional single-factor logistic regression model showed that the incidence of postoperative complications in patients with PPI was significantly greater with older age, high body mass index (BMI), smoking history, poor nutritional status, and decreased platelet counts $(\mathrm{P}<0.05)$. Results of the unconditional multivariate logistic regression analysis showed that older age, high BMI, smoking history, poor nutritional status, and decreased platelet counts were all independent risk factors of postoperative complications in patients with $\mathrm{PPI}(\mathrm{P}<0.05)$.

Conclusions: The incidence of postoperative complications in patients with PPI was low, and the complications were mainly related to capsular bag. Factors, such as older age, high BMI, smoking history, poor nutritional status, and decreased platelet counts, were independent risk factors resulting in postoperative complications in patients with PPI.
\end{abstract}

Keywords: Permanent pacemaker implantation (PPI); postoperative complications; incidence; risk factors

Submitted Aug 28, 2020. Accepted for publication Oct 06, 2020.

doi: $10.21037 /$ jtd-20-2913

View this article at: http://dx.doi.org/10.21037/jtd-20-2913

\section{Introduction}

With the development of the economy and improvements in living standards, the aging structure of China is gradually increasing. However, this brings an increase in age-related diseases, such as diabetes, hypertension, and coronary heart disease. Among these diseases, coronary heart disease is most common, which is also called coronary atherosclerotic disease. The current treatments of coronary heart disease include drug therapy, interventional therapy and surgical treatment (1). Drugs on antiplatelet, anticoagulant and lipid regulating therapy are the key to the prevention and treatment of coronary heart disease. However, the effects of antiplatelet drugs in the population are very diverse, and some patients cannot achieve sufficient degree of platelet inhibition effect after taking antiplatelet drugs, called aspirin 
or clopidogrel resistance (2). Therefore, the development of new antiplatelet drugs has been the focus of research, and ISAR-REACT 3 is one of the most interesting aspects of anticoagulant therapy for coronary heart disease. ESC and AHA/ACC/SCAI guidelines continue to regard surgical bypass grafting as standard treatment for left main disease (3). Interventional therapy is only the alternative treatment of high-risk patients who are not suitable for surgical bypass transplantation. Moreover, an increasing number of clinical trials $(4,5)$ have shown that stenting in the treatment of unprotected left main disease is comparable to surgical bypass transplantation in the near term. Coronary heart disease can have many manifestations, chronic arrhythmia is one of the common types (6). At present, there are various clinical therapies for chronic arrhythmias. The implantation of permanent cardiac pacemakers has been widely used in clinical practice because of its good treatment efficacy for severe bradyarrhythmias, partial tachyarrhythmias, and other cardiac disorders (7). With the increasing number of patients with chronic arrhythmias, the application of permanent pacemaker implantation (PPI) has gradually increased in recent years (8). In has been reported that there are about 3.25 million patients implanted with permanent pacemaker in China. However, with the increase of treatments, research has found that PPI is an invasive procedure that may make patients at risk of complications at the same time of improving the survival rate of patients (9). It has been proposed in relevant studies (10) that there will be capsular complications after PPI, but there has been no further in-depth study on why and how to effectively avoid such complications. Therefore, how to reduce the occurrence of pacemaker complications and improve the efficacy of pacemaker has attracted more and more attention from clinicians. In the present study, we discuss the incidence of postoperative complications of patients with permanent cardiac pacemaker implantation, and analyze the related risk factors of these complications, which might provide further guidance for preventing PPI postoperative complications and improving the prognosis of patients. We present the following article in accordance with the STROBE reporting checklist (available at http:// dx.doi.org/10.21037/jtd-20-2913).

\section{Methods}

\section{Patients}

A total of 124 patients who underwent permanent cardiac pacemaker implantation in our hospital from January 2014 to January 2018 were selected for participation in the present study. The patients included 74 males and 54 females, aged 30-86 years (mean: 64.28 \pm 5.08 years).

The inclusion criteria were as follows: (I) patients $>20$ years; (II) patients who met the criteria of chronic arrhythmia according to the 1998 American College of Cardiology/American Heart Association (ACC/AHA) and the 2002 Chinese Medical Association's electrophysiology, and the PPI standard in the implantable device guide of the annual meeting (11). The exclusion criteria were as follows: (I) patients with PPI contraindications; (II) patients who cannot finish the operation; (III) patients have other heart diseases besides chronic arrhythmia; (IV) patients with malignant tumors; (V) patients with primary mental disorders; and (VI) patients with incomplete or inaccurate clinical data. This study was approved by Affiliated Hospital of Zunyi Medical University (No. [2016]2-103). All procedures performed in this study involving human participants were in accordance with the Declaration of Helsinki (as revised in 2013). Individual consent for this retrospective analysis was waived.

\section{Data collection and operation methods}

The clinical data of all patients were retrospectively analyzed. For the PPI preparation, all patients underwent subclavian vein puncture using accepted implantation methods; pacemaker lead wires were then implanted and the pacemaker was implanted with a single incision on the puncture side. The implanted pacemakers were singlechamber pacemakers and dual-chamber pacemakers. All patients underwent electrocardiogram monitoring during the perioperative period. The parameters of the electrode lead were tested during the operation. After operation, the puncture site was compressed by sandbags for 6- $8 \mathrm{~h}$, and the patients were instructed to rest in the supine position for 24-48 h. Antibiotics were used for 3 days to prevent infection. The stitches were generally removed on postoperative day 7 .

\section{Clinical data}

Clinical data, such as sex, age, disease status, and incidence of related postoperative complications, were recorded for all patients, and the differences were compared to analyze the relevant risk factors affecting the incidence of complications following PPI. 


\section{Statistical analysis}

All data were analyzed by SPSS version 18.0. Normally distributed data were presented as mean \pm standard deviation. Count data were presented as the adoption rate and composition ratio, and analyzed by $\chi^{2}$-test. Unconditional univariate and multivariate logistic regression models were used to analyze the related risk factors affecting the postoperative complications of patients with PPI. $\mathrm{P}<0.05$ was considered to be statistically significant.

\section{Results}

\section{Postoperative complications of patients with PPI}

Based on the clinical data, the complication rate of the 124 patients with PPI was $8.06 \%$ (10/124), including 4 cases $(3.23 \%)$ with cystic hematoma, 2 cases with cyst rupture (1.61\%), 3 cases with capsular infection (2.42\%), and 1 case with venous thrombosis $(0.81 \%)$.

\section{Single-factor analysis of postoperative complications in patients with PPI}

Unconditional univariate logistic regression model analysis showed that the incidence of postoperative complications of PPI significantly increased in patients who were older and had high body mass index (BMI), a history of smoking, poor nutritional status, and reduced platelet counts than those who were younger, with a normal BMI, no smoking history, good nutritional status, and normal platelet counts $(\mathrm{P}<0.05)$ (Table 1).

\section{Multifactor analysis of postoperative complications in patients with PPI}

Based on the unconditional multivariate logistic regression model analysis, older age, high BMI, smoking history, poor nutritional status, and low platelet counts were all independent risk factors that led to postoperative complications in patients with PPI $(\mathrm{P}<0.05)$ (Table 2$)$.

\section{Discussion}

The permanent artificial cardiac pacemaker performs artificial cardiac pacing through implantation of longterm endocardial electrodes and pulse generators, which is the most effective treatment for irreversible dysfunction of cardiac pacing or conduction. However, because this treatment is still an invasive treatment, the incidence of complications cannot be prevented. According to previous clinical data, related complications, such as capsular bag and blood vessel problems, are common in patients undergoing PPI. If these complications are not treated in a timely manner, the treatment efficacy and prognosis of PPI in patients might be affected, along with the progression of the disease. Therefore, PPI complications have become a focus of research among cardiovascular physicians $(12,13)$. Relevant data have previously shown that the postoperative complication rate of patients with PPI is approximately $8.2-17.4 \%$. The results of the present study showed that the postoperative complication rate of the enrolled 124 patients with PPI was $8.06 \%$ (10/124), which was similar to the findings a previously published study (14).

The data of the present study showed that patients with PPI who were older, and had a high BMI, a history of smoking, poor nutritional status, and reduced platelet counts had significantly greater postoperative complications than those who were younger, with no smoking history, normal BMI, good nutritional status, and normal platelet counts. Previous clinical studies have reported that incomplete intraoperative hemostasis, hemorrhage of small arteries in the sac cavity, improper size of the capsular bag, and multiple punctures can all increase the risk of postoperative cystic hematoma. on the other hand, the use of anticoagulant drugs and older age can both lead to an increased risk of cystic hematoma among patients. Studies have shown that excessive use of heparin and the combined use of heparin with aspirin can result in a greater incidence of cystic blood lipids (15), indicating that the discontinuation of anticoagulant drugs before surgery has important clinical significance for patients. The findings of the present study also showed that the incidence of complications was significantly greater in patients who were older or who used anticoagulant drugs, which is similar to previous reports. Therefore, among the patients treated with permanent cardiac pacemaker implantation, the patients receiving anticoagulant therapy must stop using anticoagulant drugs for at least $3 \mathrm{~d}$ before the pacemaker implantation surgery and lasting until 7-10 d after the operation, so that the prothrombin time and hemostasis time of the patients can reach normal. One previously published study demonstrated that that poor nutritional status and more times of operation could further lead to the incidence of capsular rupture in patients, suggesting that they both are risk factors for this complication (16), and this 
Table 1 Single-factor analysis of postoperative complications in patients with permanent pacemaker implantation

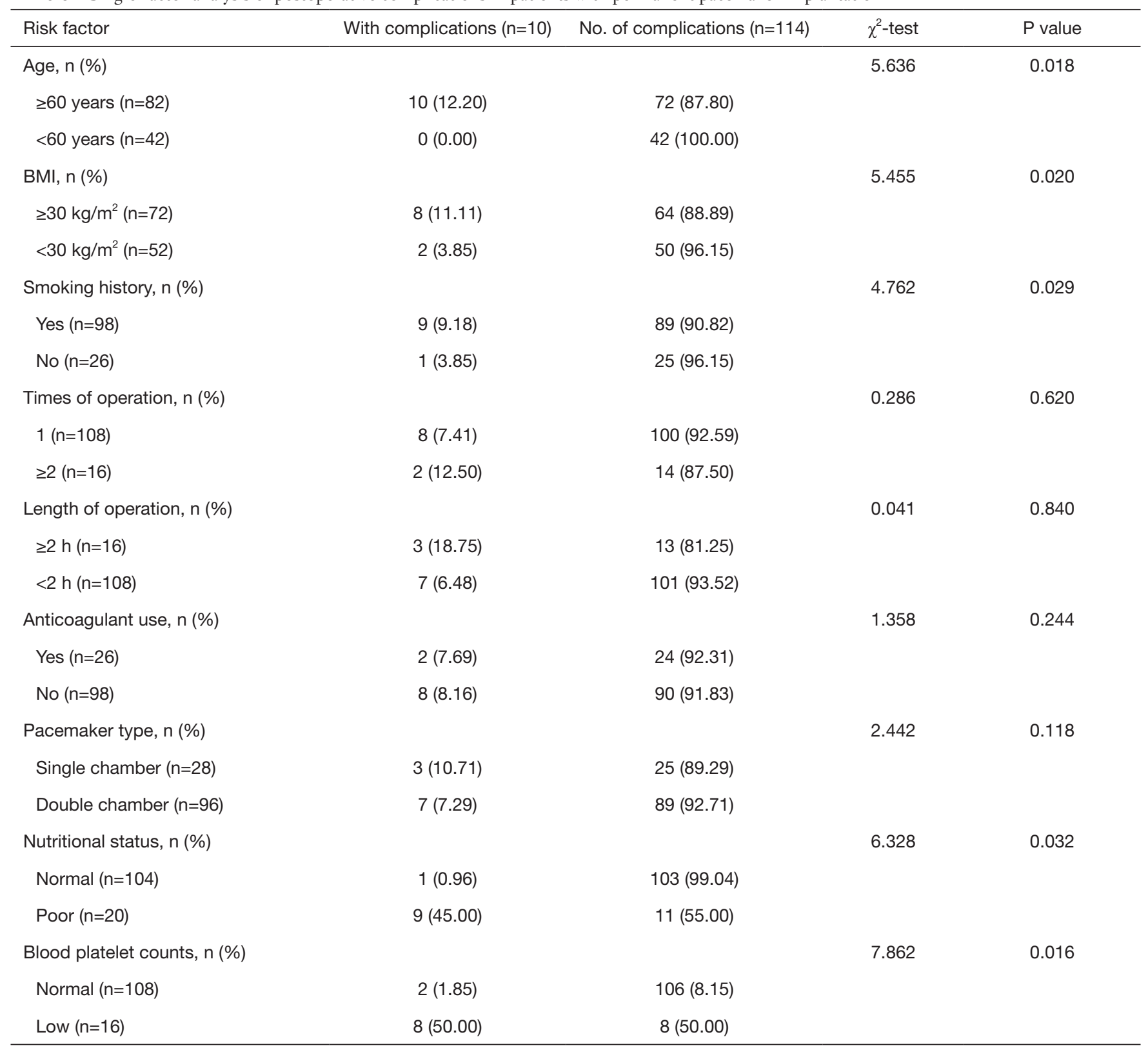

BMI, body mass index.

conclusion is also confirmed by this study. The mechanism underlying this phenomenon may be that, as nutritional status decreases, resistance of patients to some diseases is significantly reduced. Due to the shallow position of the capsular bag under the skin, when nutritional status is poor, or there are comorbidities, the blood supply of the skin may be affected to a certain extent, leading to the poor prognosis of the capsular bag. For elderly patients, the aging of the blood vessel wall is a concern, as blood rheology is subject to change. In addition, elderly patients are more likely to have hypercoagulability to varying degrees and to be bedridden after the operation, which will lead to poor blood circulation, and result in postoperative complications, such as venous thrombosis. In a previously published study, it was shown that the risk of venous thrombosis increases with age $(17,18)$, suggesting that for the elderly patients 
Table 2 Multifactor analysis of postoperative complications in patients with permanent pacemaker implantation

\begin{tabular}{lccccc}
\hline Risk factor & Regression coefficient & SEM & Wald $\chi^{2}$-test & P value & Hazard ratio (95\% Cl) \\
\hline Age (years) & 0.530 & 0.208 & 4.242 & 0.039 & $1.70(1.13-2.55)$ \\
BMI & 0.624 & 0.228 & 4.419 & 0.036 & $1.87(1.19-2.92)$ \\
Smoking history & 0.621 & 0.302 & 4.113 & 0.043 & $1.86(1.03-3.36)$ \\
Nutritional status & 0.568 & 0.256 & 4.402 & 0.036 & $1.76(1.07-2.91)$ \\
Blood platelet counts & 0.762 & 0.336 & 7.333 & 0.007 & $2.14(1.11-4.14)$ \\
\hline
\end{tabular}

$\mathrm{BMI}$, body mass index; $\mathrm{Cl}$, confidence interval; SEM, standard error of mean.

who undergo PPI, blood flow should be closely monitored and appropriate activities should be suggested to avoid complications, such as venous thrombosis. The blood of obese patients is relatively viscous. Excessive pressure in the right heart reduces the blood flow, leading to blood stasis and thrombosis. Age, obesity, smoking history, diabetes mellitus, blood hypertension, hypertriglyceridemia, and metabolic syndrome are all risk factors for PPI complications. Smoking $>15$ cigarettes a day increases the relative risk of venous thrombosis by 2.82 -folds than that of non-smokers (19). The increased oxidation of low-density lipoprotein in patients who smoke leads to an increase of blood viscosity and coagulability, and an increased risk of thrombosis (20). The findings of this study also indicated that older age, high BMI, smoking history, poor nutritional status, and low platelet counts were independent risk factors for postoperative complications in patients with PPI, suggesting that for patients who are elderly, obese, have a smoking history, poor nutrition status, or low platelet PPI, patients should have regular monitoring, with drugs administered to reduce the incidence of postoperative complications and improve prognosis. Therefore, it is recommended to take the following effective measures for the patients treated with PPI: (I) stop using anticoagulant and anti-platelet drugs for more than $10 \mathrm{~d}$ before pacemaker implantation, and treat the patients with decreased platelet count and abnormal coagulation indexes, and delay pacemaker implantation if necessary; (II) Preoperative evaluation of elderly patients and malnourished patients should be strengthened; (III) Timely find and relieve the complications to avoid aggravating the impact on the prognosis of patients.

In summary, the incidence of postoperative complications in patients undergoing PPI is low, and the major complications are related to capsular bag. Factors, such as older age, high BMI, smoking history, poor nutritional status, and low platelet counts are all independent risk factors for postoperative complications in patients with PPI.

\section{Acknowledgments}

Funding: None.

\section{Footnote}

Reporting Checklist: The authors have completed the STROBE reporting checklist. Available at http://dx.doi. org/10.21037/jtd-20-2913

Data Sharing Statement: Available at http://dx.doi. org/10.21037/jtd-20-2913

Conflicts of Interest: All authors have completed the ICMJE uniform disclosure form (available at http://dx.doi. org/10.21037/jtd-20-2913). The authors have no conflicts of interest to declare.

Ethical Statement: The authors are accountable for all aspects of the work in ensuring that questions related to the accuracy or integrity of any part of the work are appropriately investigated and resolved. This study was approved by Affiliated Hospital of Zunyi Medical University (No. [2016]2-103). All procedures performed in this study involving human participants were in accordance with the Declaration of Helsinki (as revised in 2013). Individual consent for this retrospective analysis was waived.

Open Access Statement: This is an Open Access article distributed in accordance with the Creative Commons Attribution-NonCommercial-NoDerivs 4.0 International License (CC BY-NC-ND 4.0), which permits the noncommercial replication and distribution of the article with 
the strict proviso that no changes or edits are made and the original work is properly cited (including links to both the formal publication through the relevant DOI and the license). See: https://creativecommons.org/licenses/by-nc-nd/4.0/.

\section{References}

1. Villa E, Clerici A, Messina A, et al. Risk Factors for Permanent Pacemaker after Implantation of Surgical or Percutaneous Self-Expanding Aortic Prostheses. J Heart Valve Dis 2016;25:663-71.

2. Kirolos I, Ifedili I, Maturana M, et al. Ticagrelor or prasugrel vs. clopidogrel in combination with anticoagulation for treatment of acute coronary syndrome in patients with atrial fibrillation. Ann Transl Med 2019;7:406.

3. Polikandrioti M, Tzirogiannis K, Zyga S, et al. Assessment of fatigue in patients with a permanent cardiac pacemaker: prevalence and associated factors. Arch Med Sci Atheroscler Dis 2018;3:e166-73.

4. Aronow WS, Banach M. Atrial Fibrillation: The New Epidemic of the Ageing World. J Atr Fibrillation 2009;1:154.

5. Belardinelli R, Capestro F, Misiani A, et al. Moderate exercise training improves functional capacity, quality of life, and endothelium-dependent vasodilation in chronic heart failure patients with implantable cardioverter defibrillators and cardiac resynchronization therapy. Eur J Cardiovasc Prev Rehabil 2006;13:818-25.

6. Afzal MR, Savona S, Mohamed O, et al. Hypertension and Arrhythmias. Heart Fail Clin 2019;15:543-50.

7. El-Assaad I, Al-Kindi SG, Oliveira GH, et al. Pacemaker implantation in pediatric heart transplant recipients: Predictors, outcomes, and impact on survival. Heart Rhythm 2015;12:1776-81.

8. Mohananey D, Jobanputra Y, Kumar A, et al. Clinical and Echocardiographic Outcomes Following Permanent Pacemaker Implantation After Transcatheter Aortic Valve Replacement: Meta-Analysis and Meta-Regression. Circ Cardiovasc Interv 2017;10:e005046.

9. Moskowitz G, Hong KN, Giustino G, et al. Incidence and Risk Factors for Permanent Pacemaker Implantation Following Mitral or Aortic Valve Surgery. J Am Coll Cardiol 2019;74:2607-20.

10. Leyva F, Qiu T, McNulty D, et al. Long-term requirement for pacemaker implantation after cardiac valve replacement surgery. Heart Rhythm 2017;14:529-34.

11. Kazmirczak F, Chen KA, Adabag S, et al. Assessment of the 2017 AHA/ACC/HRS Guideline Recommendations for Implantable Cardioverter-Defibrillator Implantation in Cardiac Sarcoidosis. Circ Arrhythm Electrophysiol 2019;12:e007488.

12. Dipoce J, Bernheim A, Spindola-Franco H. Radiology of cardiac devices and their complications. Br J Radiol 2015;88:20140540.

13. de Oliveira GB, de Oliveira FS, Osório AP, et al. Late Outcome and Predictors of Adverse Events Related to the Implantation of a Permanent Pacemaker in Patients with Isolated Congenital Atrioventricular Block. Pediatr Cardiol 2016;37:1319-27.

14. Silva KR, Albertini CM, Crevelari ES, et al. Complications after Surgical Procedures in Patients with Cardiac Implantable Electronic Devices: Results of a Prospective Registry. Arq Bras Cardiol 2016;107:245-56.

15. Elmahashi MO, Elbareg AM, Essadi FM, et al. Low dose aspirin and low-molecular-weight heparin in the treatment of pregnant Libyan women with recurrent miscarriage. BMC Res Notes 2014;7:23.

16. Lidegaard Ø. Hormonal contraception, thrombosis and age. Expert Opin Drug Saf 2014;13:1353-60.

17. Petousis V, Sallam AA, Haynes RJ, et al. Risk factors for retinal detachment following cataract surgery: the impact of posterior capsular rupture. Br J Ophthalmol 2016;100:1461-5.

18. Prandoni P. Venous and Arterial Thrombosis: Is There a Link? Adv Exp Med Biol 2017;906:273-83.

19. Anand SS. Smoking: A Dual Pathogen for Arterial and Venous Thrombosis. Circulation 2017;135:17-20.

20. Aslian H, Kron T, Longo F, et al. A review and analysis of stereotactic body radiotherapy and radiosurgery of patients with cardiac implantable electronic devices. Australas Phys Eng Sci Med 2019;42:415-25.

(English Language Editor: R. Scott)

Cite this article as: Jing S, Hu S, Ma S. Analysis of postoperative complications and risk factors in patients with permanent pacemaker implantation. J Thorac Dis 2020;12(10):5980-5985. doi: 10.21037/jtd-20-2913 